\title{
Design of Wireless Remote High-voltage Energy Metering Systems
}

\author{
Meng Aini \\ College of Automotive Engineering,Weifang University of Science and Technology \\ Shouguang , 262700,China \\ email: 377605208@qq.com
}

Keywords: High-voltage energy acquisition. Wireless transmission. ATT7022

\begin{abstract}
Articles on energy metering performed via remote communications platform to explore new technologies and designed measurement system software, hardware and implementation methods.Energy metering system design includes selection of components, hardware design and software programming and debugging.This paper presents the hardware design of the high-voltage system as a whole can be measured overall flow chart diagram and software, and around the main function of the hardware devices and software were compared in detail. The system is a high anti-jamming capability, accuracy is strong, stable data transmission, the use of good results in the project.
\end{abstract}

\section{Introduction}

In the measurement of user accounts and the electricity sector, the measurement results of energy metering device is an important basis for the statistical indicators in energy usage and as an important basis for settlement.High-voltage energy metering system for a long time in the past generally use the table with metering, meter reading artificial way,which impedes the pace of power system automation and intelligent development.Currently, the high-voltage energy metering device of 10kV Distribution Network in the extensive use of basic equipment consists of a combination of electric current transformers (Current Transformer), voltage transformer (Potential Transformer) and smart meter and so on.Since the current and voltage transformers, and smart energy meter itself, there are some errors in the work process, thus leading to a high-voltage electrical energy metering device in the measurement of the accuracy is not high.High-pressure measurement system error is generally generated by the component itself, but also affected by factors such as transformer secondary voltage drop.

\section{High-voltage Energy Metering Principle}

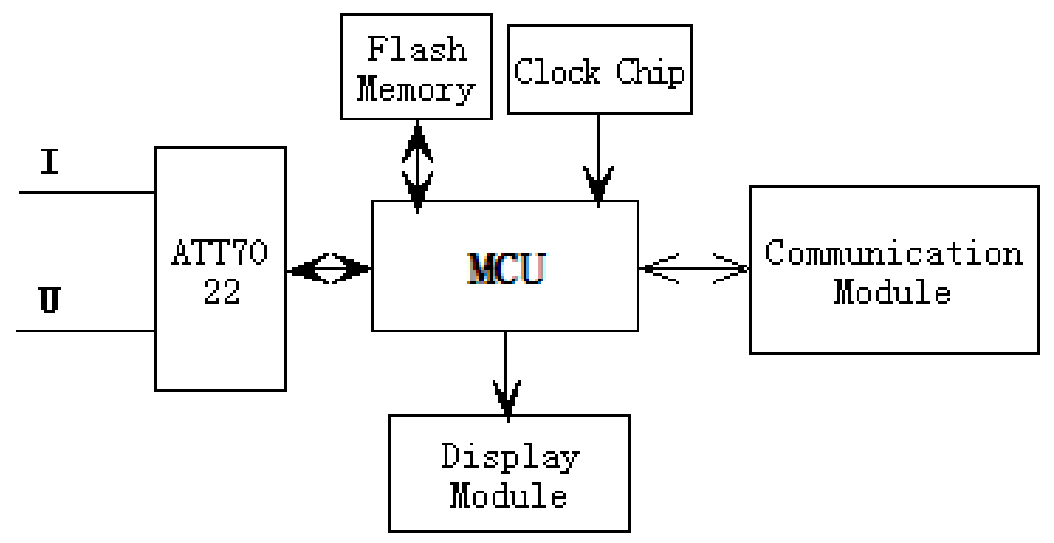

Fig.1 The total design of measurement system

High-voltage energy metering system consists of a current transformer (CT), voltage transformer (PT), acquisition module, smart meter and a secondary wire that connects them with the 
communication system and so on.These intermediate component parts of any failure will cause an inaccurate measurement result, and even lead to paralysis of the entire high-pressure measurement system ${ }^{[1]}$.The high-pressure metering system design in terms of data transmission mainly adopt stable GPRS wireless communication technology, and using the latest technology with high precision three-phase power metering chip dedicated ATT7022, the overall design of the energy measurement system block diagram shown in Figure 1 .

\section{The Hardware Designing of Metering System}

Microcontrollers. The microprocessor is the core part of the metering system, the system uses microprocessor is STM32F103VBT6 chip ${ }^{[2-3]}$.STM32F103VBT6 chip is ARM's latest high-speed, low-power 32-bit microcontroller, using the Cortex-M3 core, the operating frequency multiplier up to $72 \mathrm{MHz}$, the ability to provide low-voltage operation of $2.0 \mathrm{~V}-3.6 \mathrm{~V}$ when using the battery.The chip also has a dual structure allows for dual-channel ADC sample / hold to achieve 12-bit accuracy.In addition, a plurality of communication interfaces including two I2C interface (400KHZ), 3 个 USART synchronous asynchronous serial interface (4.5Mbit / s), 2 two SPI synchronous serial interface (18Mbit / s), CAN interface and USB interface each a. On the function and structure are designed to meet the hardware requirements of the system.

Energy Metering IC. This paper uses a huge energy metering module Springs Optoelectronics Technology production ATT7022E chip, which is a versatile, high-precision measurement of three-phase power dedicated chip, for three-phase three-wire and three-phase four-wire applications.The chip integrates seven second-order sigma-delta ADC, voltage reference circuit and all the power, energy, RMS, digital signal processing power factor and frequency measurements of other circuits, capable of measuring each phase and phase combined active power, reactive power, apparent power, active energy and reactive energy, while measuring the phase current, RMS voltage, power factor, phase angle, frequency and other parameters to fully meet the needs of multi-rate multi-functional three-phase energy meter .ATT7022 chip uses a single $+3.3 \mathrm{~V}$ supply, the structure provides an SPI interface for easy connection to the microprocessor, and increase the speed of data transmission, SPI bus inevitably receive high-voltage lines and other external interference in the transmission of environmental data, the microprocessor is connected communication lines should be as short as possible, and copper.When high-frequency signals to eliminate the interference can be a small resistor in series with the SPI bus signal line and the input signal is applied at one end of the set of decoupling capacitors. ATT7022 peripheral circuit design shown in Figure 2.

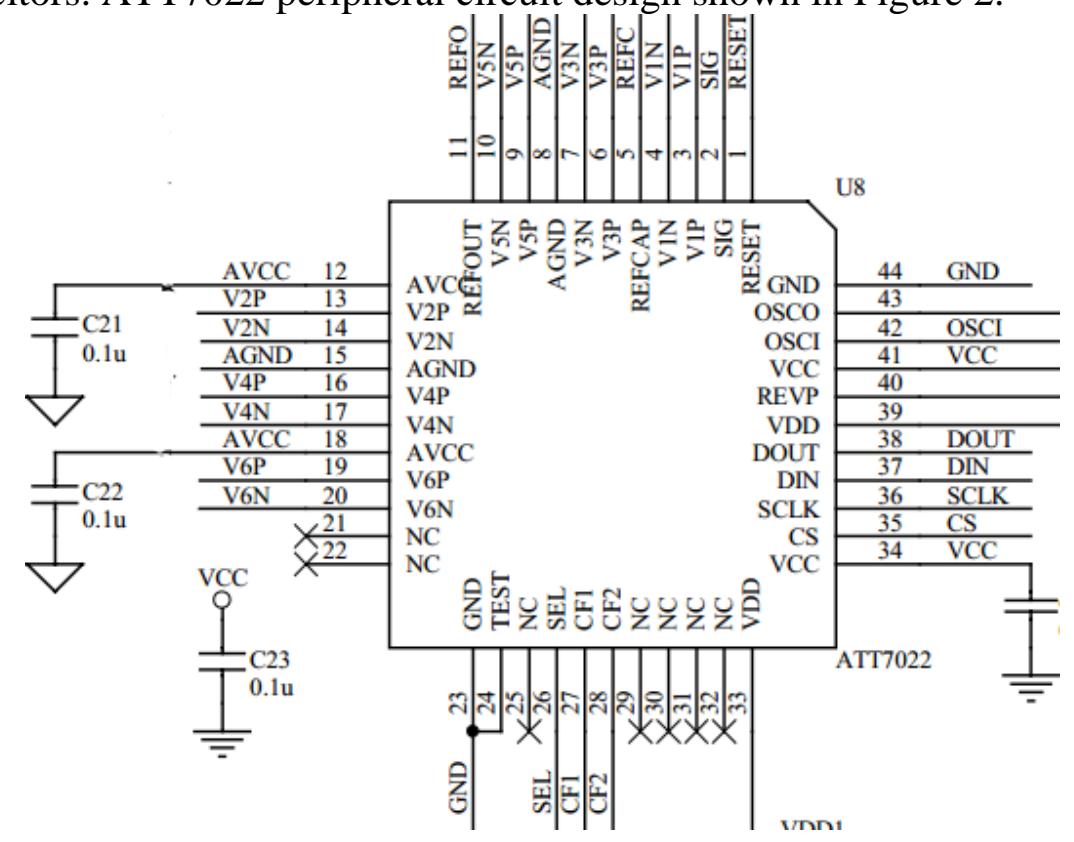

Fig.2 ATT7022 Peripheral Circuit 
Clock circuit. When you first install energy metering system, first read after power network time clock chip communication module and write time during system operation, the read time of day clock chip and with reference to the network time, the difference between hope when more than $5 \mathrm{~min}$, the correction to the network based on the time clock chip time.

The clock module is selected DALLAS company's calendar clock chip DS12C887 clock chip that has a long record calendar information, and measuring system failures due to power-down the next line time clock chip information can also be in for a long time there does not appear to stop timing, etc., and can generate clock interrupts.

The chip has 128 bytes of RAM, which includes 114 bytes of general purpose RAM. This can save the measurement values are stored for each chip, including metering and data after power is not easily lost.

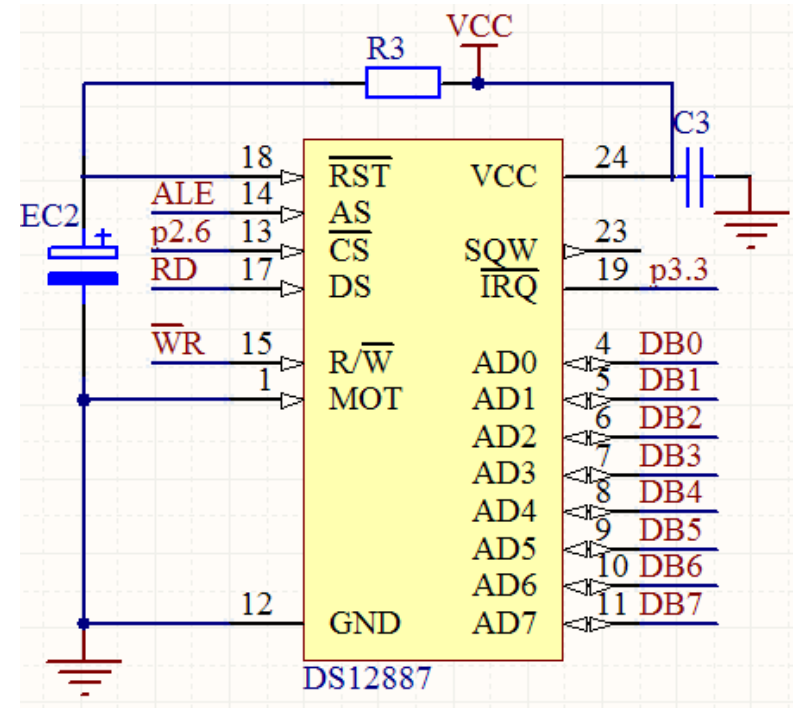

Fig.3 Clock circuit diagram

$3 G$ wireless communication circuit. EM770W Huawei company's $3 \mathrm{G}$ wireless communication module, supports WCDMA, widely used in the terminal equipment and industrial fields, such as mobile phones, wireless meter reading, wireless monitoring.In HSPA, the maximum download speed of its uplink $5.76 \mathrm{Mbps}$, download rate downlink 7.2Mbps, with low power consumption, good compatibility, transmission speed, stability and other characteristics.EM770W addition to built-T CP / IP protocol stack, it also supports the AT command set that provides high-speed data transmission services for the remote monitoring system ${ }^{[4]}$.

The energy metering system to communicate with the host computer database. There are three main forms: When GPRS module receives a read command sent by the user to copy, promptly return the real-time energy metering values; when regular meter reading time, the metering system will cycle through the communication platform upload measurement value; when an accident happens, the metering system to send alarm information to the user via a communication platform.

\section{The software designing of metering System}

Part of high-voltage energy metering system software mainly consists of GPRS module driver, energy metering data transmission and preservation, alarms, etc. ${ }^{[5]}$.

When normal power metering system, first initialization settings, including the time proofreading, GPRS networking, after the end of the metering system initialization command is received to determine whether to send a text message untreated, and then measure the chip for data transmission and save, when data do not meet the limit values set by the metering system will send alarm information to the user via a communication platform. Overall software design flow chart shown in Figure 4. 


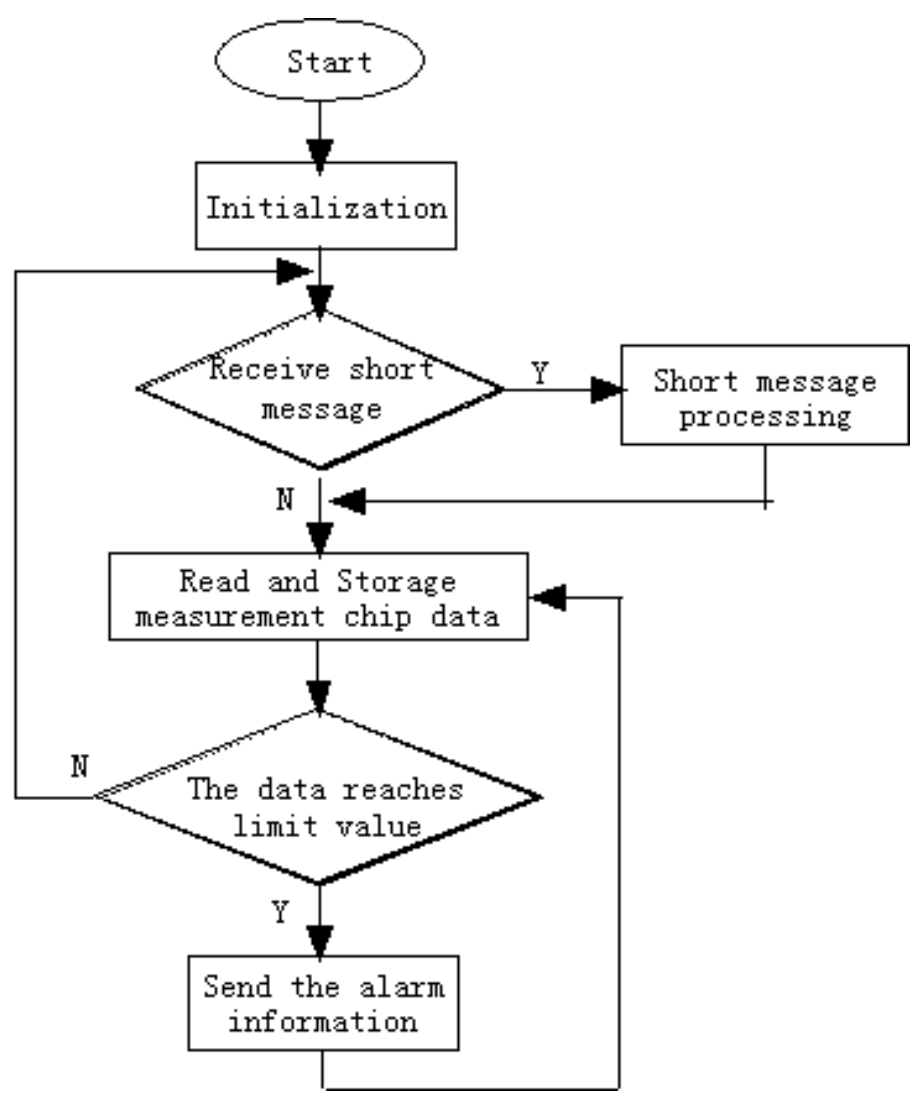

Fig.4 Overall Flowchart Software

\section{Summary}

Energy metering system is the bridge power system and user connections, energy metering system via a wireless network users and data analysis grid and effective integration platform as a whole.In this paper, the programming STM32F103VBT6,3G communication module, energy measurement chip ATT7022C hardware circuit design and software design of the final completion of the entire system. Through internal testing, the experimental measurement data errors within the allowed range. The system data acquisition speed, low power consumption, stable operation, and achieve the desired design goals of the system. The system can be used in remote power systems, electric power system or the like mine precision measurements.

\section{References}

[1] Zhao Jianjun,Zhang Sujun. Fault Analysis and Modeling for the High Voltage Electric Power Metering System[J]. Electrical Measurement \& Instrumentation, 2007; 44(4):5-8.

[2] STM32F10xxx_Reference_Manual,www.st.com

[3] Li Ning. MDK-based development and application of the STM32 processor [M]. Beihang University Press. 2008: 11-13.

[4] Yang H T, Eagleson R. Design and Implementaion of an Internet-based Embedded Control System.Proceedings of 2003 IEEE Conference on Control Applications, Istanbul, 2003, 2:1181-1185.

[5] Zhang Delong, The Design of Remote Electric Energy Collection and Control Device for High Voltage Based on GPRS[D]. Baoding: Agricultural University of Hebei. 2012 . 